Patrycja Polanowska

Università di Varsavia

Dipartimento di Italianistica

https://doi.org/10.18778/8220-478-0.03

\title{
POESIA DELLA “DISTRUZIONE COMPIUTA” MILO DE ANGELIS E LA SUA RADICE DELL'ITALIANITÀ
}

\begin{abstract}
Questo articolo traccia degli aspetti dell'italianità nell'opera di Milo De Angelis, che esordisce nel 1976 con Somiglianze. La sua poesia, opponendosi alla crisi poetica del secondo Novecento, cerca di rifondare lo statuto del discorso lirico, restando pur sempre profondamente radicata nella corporalità delle cose. Le seguenti analisi vengono focalizzate su due punti principali, esaminati tramite i riferimenti alla letteratura italiana. Il primo di essi è quello della rappresentazione di Milano, in cui vengono scoperti degli influssi della poetica di Charles Baudelaire che permettono a De Angelis di abbandonare l'orizzonte usuale della sua città. La peculiarità rilevata nella seconda parte del saggio è il carattere della parola poetica in sé, determinata da un contrasto interno tra l'elemento dionisiaco ed apollineo.
\end{abstract}

Parole chiave: poesia contemporanea, crisi, sublime, Milano, mito

Abstract: This article aims to trace some aspects of Italianness in the oeuvre of Milo De Angelis, debuting in 1976 with Somiglianze. Despite the crisis in poetry in the second half of the twentieth century, his writing, profoundly rooted in the corporeality of things, attempts to restore the status of lyrical discourse. The analyses are focused on two principal points, examined through some references to Italian literature. The first of them is the representation of Milan, that turns out to be influenced by poetics of Charles Baudelaire, what helps De Angelis to abandon the usual horizon of his city. A peculiarity highlighted in the second part of the paper is the character of a poetic word itself, determined by an inner contrast between the Apollonian and the Dionysian.

Keywords: contemporary poetry, crisis, sublime, Milan, myth 
«Non mi creda così pura. A volte ho degli incubi. Mi rifugio nei bar del mio rione, in Viale Monza» $\ll \ldots \gg$ $\ll$ Ma adesso non mi creda vile. Anche quando soffro... anche quando ho paura sono la bambina che scopre il temporale e gli va incontro da sola $\gg^{1}$.

Il frammento citato, che conclude gli appunti di Aprile 1980 messi nell'ultima sezione del volume di saggi teoretici di Milo De Angelis, intitolato Poesia e destino, può essere visto come nota (auto)riassuntiva della sua poesia. Questa è una lirica fatta di silenzi, squarci e ferite, rapita dall'oscurità del mito e avviata da un dialogo impossibile e un contemporaneo susseguimento delle cose che non appartengono all'ordine in cui siamo abituati a collocarle. Le premesse ideologiche che ne stanno alla base, come afferma Giorgio Bàrberi Squarotti, “risentono di una cultura del 'negativo' e dello scacco e del 'perdersi' di ascendenza francese, fra Sartre e Blanchot e Bataille, con Rimbaud alle spalle"2. Le schegge della sua immagine del mondo ci rimandano alle borgate milanesi dove un dissidio interno tra materia e vuoto viene ratificato e contemporaneamente reso ancora più intenso. L'eterno presente dell'opera deangelisiana, quello spazio della solitudine retta dalla distanza che abbiamo davanti e dietro, non sfocia nella purezza artistica, ma risulta essere il luogo della tragedia e del contemporaneo "disprezzo di un rimedio" ${ }^{3}$. Il suo cronotopo viene circoscritto al perimetro della parola, una parola capace di superare la realtà senza tuttavia trascenderla. La tensione all'altrove pare essere sostituita da un eterno ritorno e così, il tentativo di arrivare all'essenza delle cose non consiste nella riduzione dei loro elementi accidentali (come avviene ad esempio nel caso di Mallarmé), ma risiede nel secondo sguardo che un io lirico concede ad oggetti e fenomeni percepiti.

De Angelis, in uno spirito che risale alle tesi di Blanchot e Leopardi, situa la propria poesia in un momento soglia dove non succede di

avere paura, quanto piuttosto di diventare noi stessi paura, nel sangue che scorre più veloce, nei battiti che aumentano: questa paura da cui non vogliamo più sottrarci è la scoperta del corpo sconosciuto: lì, fin dall'inizio, era nato il tutto che non ci appartiene e che continua a parlarci oltre la finestra ${ }^{4}$.

1 M. De Angelis (1982), Poesia e destino, Bologna, Cappelli, p. 140.

2 G. Barberi Squarotti (1975), Introduzione a "Lidea centrale", Almanacco dello Specchio, 4, p. 375.

3 M. De Angelis, op. cit., p. 41.

4 Ibidem, pp. 102-103. 
Tale esposizione all'indeterminato, non porta però alla seguente cancellazione dell'io e di tutte le coordinate dello spazio e del tempo, perché queste permettono il ritorno alla tradizione e ai grandi antecedenti letterari e filosofici. In quest'ottica anche l'essenza poetica delle cose non può essere scovata se non tramite l'esistenza effettiva della voce artistica. Queste dinamiche ci fanno avvicinare al nodo dell'italianità deangelisiana, un'italianità insolita, a volte anche paradossale, che sembra procedere per termini di distacco, ritorno e apertura.

Il nostro autore, già dai tempi della sua raccolta d'esordio, Somiglianze (1976), si colloca in contrapposizione agli approcci dominanti nella poesia italiana dell'epoca, da una parte relativi alle tendenze sperimentali proprie del Gruppo 63, e dall'altra segnati dalle appartenenze ideologiche militanti. Seguendo lo spirito della lirica moderna, De Angelis vede il senso ultimo della poesia nella parola stessa e non nella liberazione dal dolore che l'esperienza artistica continua a procurare. I suoi componimenti, retti da uno sfondo esistenziale, dalle forze del niente e della solitudine, paiono essere assediati dal dramma dell'impossibilità di dire, con estrema precisione, ciò che non è preciso. A saldare questo taglio poetico contribuisce il riconoscimento delle vicinanze con gli autori come Leopardi, Montale (delle Occasioni e della Bufera), Pavese, Campana, Michelstaedter, nonché con quelli che De Angelis definisce come suoi veri e propri maestri, ovvero Franco Fortini, Piero Bigongiari e Mario Luzi. In forza di questi riferimenti, la parola deangelisiana punta a recuperare tutto ciò che il pensiero e i sensi non trovano nel mondo circostante: mantenendo un'immanente tensione verso il sublime, la parola viene afferrata "nell'attimo della sua rivelazione apocalittica" e più vera del reale, si merita di rimanere non spiegata.

Il cronotopo lirico di De Angelis, come abbiamo già osservato, viene innestato nella realtà della Milano contemporanea: a questo livello, che per intuizione sembra essere un aspetto edificante della italianità dell'autore, non ritroviamo nessun tratto della linea di Anceschi - fondata sulla "città simbolo di uno spirito lombardo inteso come produttività, razionalismo, illuminismo e ironia". È possibile individuarvi soltanto la periferia, fatta "di tangenziali e fabbriche abbandonate, di vicoli bui e fantasmi"7. Tale contrapposizione sembra ripercorrere le dinamiche di una grande svolta nel rappresentare la città, la cui immagine moderna sorge dal crollo del pensiero simbolico e stereotipato, del tentativo di "percepirel'assoluto attraverso la semplicelettura

\footnotetext{
5 A. Baldacci (2014), La necessità del tragico, Massa, Transeuropa, p. 91.

6 Ibidem, p. 67.

7 Ibidem.
} 
della figura del mondo"8. Nella nuova realtà, la conoscenza, come afferma Yves Bonnefoy nel suo saggio Parigi in poesia, "non aiuta più a incontrare l'essere". Così anche il principio, l'obiettivo della poesia non sono più il noto, ma piuttosto l'ignoto. Eppure, per arrivare ad esso, la poesia deangelisiana non si ritira nell'ambito della natura, fonte ispiratrice dell'artista - da Hölderlin a Nerval: è la sua Milano periferica ad originare un movimento centripeto della produzione poetica, assumendo il ruolo di spazio urbano che accoglie ed assume i tratti di altre città significative per l'autore, come Atene, Sparta, SaintNazaire o Varsavia. Esse sono segnate da una drammatica comunione delle sorti, marcate dalla presenza di un inesorabile ciclo di crollo e ricostruzione.

A questo punto bisogna rilevare che la poetica del nostro autore risulta essere fortemente segnata dalla tradizione letteraria francese, soprattutto dagli autori del secondo Ottocento, i cui testi De Angelis, grazie alla sua formazione bilingue, legge già da adolescente nella versione originale. Un fattore decisivo sono anche gli influssi degli autori d'oltralpe che riscontriamo nelle poetiche delle già citate figure centrali della Weltanschauung deangelisiana: a tale preposito basta ricordare gli studi critici di Luzi o Bigongiari. Focalizzando le nostre analisi sulla visione della città, riconosciamo nella poetica di Charles Baudelaire una delle sue principali fonti d'ispirazione. Lautore milanese condivide l'approccio di Eugenio Montale che aveva esaltato la mancanza del modello baudelairiano dentro il quadro della letteratura italiana. L'opera di De Angelis segue il perimetro del capoluogo della Lombardia, che pur diverso da Parigi, riporta degli influssi dello sguardo di Baudelaire, diventando uno spazio dove il sacro sorge dal profano, mentre il movimento lirico viene azionato dalla centralità dell'incontro. In entrambi i casi, sia al livello del contenuto che dello stile, la rappresentazione segue l'approccio realistico, ma al contempo finisce per oltrepassare i limiti del realismo stesso. La Milano del nostro poeta diventa (per dirlo con le sue parole) una "città delle macerie e delle rinascite", che "appartiene alla razza delle città distrutte, delle città in cui le cose avvengono per l'ultima volta" ${ }^{10}$. De Angelis pare essere prossimo all'impostazione di Baudelaire, nei cui testi tutto compare per scomparire subito dopo, togliendo però l'aspetto figurativo a queste immagini. Milano che vi possiamo osservare, come anche "Parigi capitale del XIX secolo", diventa il luogo della perdita continua, del trapasso e della "irrimediabile caducità"

Milo De Angelis crea nei suoi componimenti un contesto topografico vero ma straniante, rigoroso ma velato. La rappresentazione si snoda lungo

8 Y. Bonnefoy (2016), Il secolo di Baudelaire, Bergamo, Moretti\&Vitali, p. 175.

9 Ibidem.

10 M. De Angelis (2008), Colloqui sulla poesia, Milano, La Vita Felice, p. 87.

11 W. Benjamin (1986), Parigi capitale del XIX secolo, Torino, Einaudi, p. 456. 
viaggi fulminei, in cui i luoghi e gli oggetti reali vengono accostati agli elementi imprecisabili che sembrano evocare l'eternità: le autostrade e i semafori si rispecchiano negli "occhi [che] non si chiudono contro le cose", "la luce di un riflettore" si accende "sopra l'acqua", "la tenda / [...] si muove / e sogna la neve" 12 . Queste immagini, provocate da una sorta di "sonnambulismo conoscitivo" ${ }^{13}$, operano all'interno di uno spazio del limite che prende l'inizio nella realtà $\mathrm{e}$ anche in essa finisce. Per un attimo, però, le cose riescono a "separarsi dai nomi" ${ }^{14}$. Con precisione matematica, il poeta sposta le qualità e gli accenti, guarda il rovescio delle immagini, traforando la loro opacità. In quella periferia milanese le cose perdono la propria identità usuale, cessano di essere segnalate all'interno dell'ordine apparente in cui cerchiamo di ritrovarle ${ }^{15}$. La componente lineare del testo viene abrogata, la lettura procede così per visioni frammentarie del mondo esteriore, tagliate da vari elementi, quali: reminiscenze e ritorni; parole rivolte ad un interlocutore sempre assente (con dei riferimenti a L'attesa, l'oblio di Blanchot) che, tranne l'ultima raccolta, resta anche fortemente interiorizzato; sequenze dialogiche di tipo luziano che - operando per scorci e accelerazioni espressive - non permettono mai una comprensione reciproca. Un vero e proprio incontro con "una passante"16 baudelairiana non avviene, anche perché il mondo del secondo Novecento, come nota Bonnefoy, smette di allinearsi al dovere che la città "mantenga l'idea di un esistenza in comune, del dialogo sempre possibile" 17 . Di conseguenza scompare anche la folla, immanente alla voce del poeta francese. La figura di flâneur, che richiedeva un'immersione in mezzo alla gente per poter definire sé stesso, passa in De Angelis verso quella di un "camminatore solitario":

Ho fatto mille escursioni - dichiara il nostro poeta - sempre da solo, in ogni quartiere di Roma e soprattutto di Milano, cercandone storia e leggenda, odori, cortili, dialetti, intonazioni della voce umana e della voce che hanno le case [...]. Camminatore solitario, dunque. Flâneur, no ${ }^{18}$.

12 M. De Angelis (2017), Tutte le poesie, Milano, Mondadori, pp. 61, 48, 36.

13 E. Affinati (1996). Patto giurato. La poesia di Milo De Angelis, Pescara, Tracce Edizioni, p. 69.

14 M. De Angelis, Tutte le poesie, p. 37.

15 Cfr. M. Cucchi (1994), Milo De Angelis, [in:] Maurizio Cucchi, Stefano Giovanardi (a c. di), Poeti italiani del secondo Novecento. 1945-1995, Milano, Mondadori, p. 889.

16 C. Baudelaire (2008), I fiori del male, Venezia, Marsilio, pp. 250-253.

17 Y. Bonnefoy, op. cit., p. 184.

18 M. De Angelis, Colloqui sulla poesia, p. 201. 
Bisogna sottolineare che in tale contesto topografico - irreale, tragico ed ultimativo, il riconoscimento vero dei luoghi arriva spesso solo a posteriori, in modo quasi impercettibile, provocato dalla necessità di un finale aggancio allo spazio e al tempo reali che costituiscono una garanzia sulla effettiva esistenza del mondo, indispensabile anche per lo stesso poi sottrarsene. De Angelis chiude le proprie visioni con l'inserimento di coordinate come: "sono le sei meno venti" oppure "andiamo allo Zara"19, quasi per "impedirsi la definitività reale dell'uscita dal tempo dell'ordine e della normalità" ${ }^{20}$, mentre la dissonanza che dirige e centralizza il flusso lirico viene ristretta al massimo, limitandosi a volte al solo presentimento e alla sua attesa, come succede nella poesia La frazione:

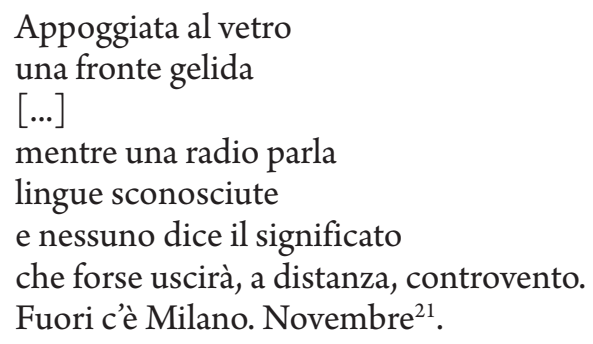

De Angelis, seguendo la poetica baudelairiana, sospende i propri versi a metà strada fra il baratro e il sublime, in un orizzonte di mistero totale. La sua parola poetica, portando in sé un'immanente tensione all'assoluto, si aggroviglia alle strade di Milano, senza immedesimarsi però con il loro aspetto figurativo. Le borgate milanesi non sono il luogo a cui questa poesia si limita, ma diventano L'oceano intorno a Milano, per citare il titolo della prima sezione della sua raccolta Biografia sommaria (1999). Quell'acqua infinita costituisce un elemento fondamentale della natura che, come i metalli ele materie minerali in Baudelaire, possiede la forza di superare l'insensibilità del mondo. In questa prospettiva, tutto si trova fuori. L'accesso alla figura di un centro viene sbarrato, ma d'altro canto quello stesso spazio, nella sua indefinitezza, costituisce il punto di transito verso brevi lampi dell'altrove e della parola che "ci precede e ci disegna"22. Essa sta sempre fuori, "oltre la finestra", inarrivabile e sempre imminente, come Milano stessa, è "lì davanti, lì davanti / come un'idea a perpendicolo / o uno sbocco di sangue / nel centimetro più lungo tra le tempie" ${ }^{23}$, sta costantemente all'ombra o rimane velata dalla nebbia, sbarrata

19 M. De Angelis, Tutte le poesie, pp. 34, 86.

20 G. Barberi Squarotti, op. cit., p. 373.

21 M. De Angelis, Tutte le poesie, p. 26.

22 M. De Angelis, Colloqui sulla poesia, p. 152.

23 M. De Angelis, Tutte le poesie, p. 249. 
(o mediata) dalle vetrine, dalla pioggia, dalla voce dal citofono. Un'espressione diventa così "un gesto / per unire i treni di Lambrate a un'antica rima" ${ }^{2}$. Il dire poetico, prima di arrivare al traguardo, si sospende proprio qui, nel passaggio che "continua a finire"; custodisce, lungo "una parabola tra le macerie" della vita reale, l'assenza di ciò che non ci è mai concesso conoscere in modo pieno, "il semprevivo di ogni niente" 25 . Proprio in questo dissidio sta il carattere tragico della poesia di De Angelis, in quell'impossibilità di perdersi nel vuoto dell'arte e una contemporanea rinuncia alla riproduzione mimetica e all'illusorietà dell'ispirazione sacrale. Lo spazio di questa parola, come afferma Giorgio Barberi Squarotti, "è quello in cui si dichiarano continuamente i momenti perduti di una vita che è prima della coscienza e che non riesce mai a porsi come autentica alternativa al discorso" 26 . Quella di De Angelis non è una poesia pura di stampo mallarmeano, essa ritrova l'assoluto tra i rottami del presente senza rivendicare una costruzione positiva dell'universo della parola. Per vedere meglio le tendenze di cui si è parlato, sembra opportuno citare un frammento della poesia Dove tutto è in relazione, proveniente dalla prima raccolta Somiglianze:

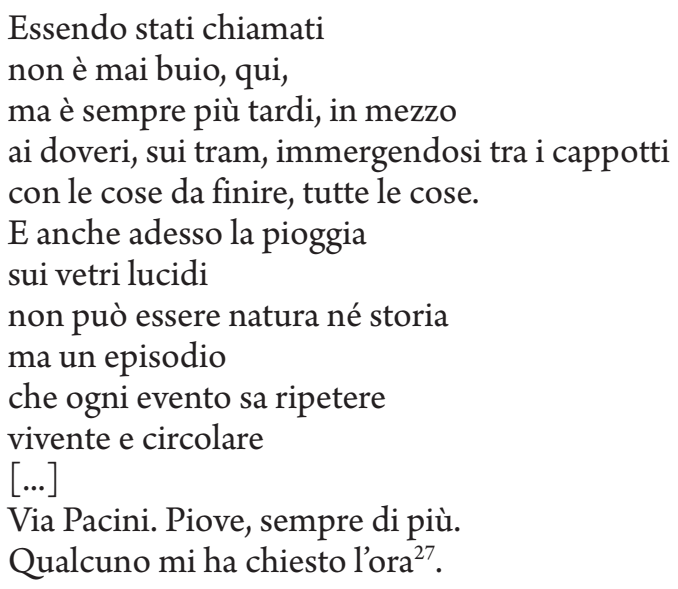

Il quadro d'alienazione dell'uomo, ritrovatosi dentro uno spazio urbano della metropoli, non può essere reso dagli strumenti tradizionali della versificazione classica volta all'armonia. Le dissonanze testuali, la sproporzione, il movimento schizofrenico e una contemporanea necessità d'ordine, esaltano gli elementi minimi del taglio orizzontale della realtà,

\footnotetext{
24 Ibidem, p. 254.

25 Ibidem, pp. 252-253.

26 G. Barberi Squarotti, op. cit., p. 373.

27 M. De Angelis, Tutte le poesie, p. 25.
} 
facendo sottrarre i versi alla rima e alle misure ritmiche. "La risoluzione del discorso in un'alternanza di sentenze e di rapidi scorci d'oggetti, di eventi, di situazioni” ${ }^{28}$ proviene proprio da quella impossibilità del testo poetico di restituire un vecchio ordine basato sull'opposizione tra sacro e profano. Non si può dimenticare che l'esordio poetico del nostro autore avviene in pieni anni Settanta, con Satura di Montale e Trasumanar e organizzar di Pasolini alle spalle e con l'esplosione di Castelporziano prossima a venire. Anzi. Le poesie di De Angelis vengono pubblicate nell'antologia di Berardinelli e Cordelli, Il pubblico della poesia, dove nel famoso brano di Effetti di deriva, Berardinelli denuncia il "fenomeno di sgretolamento di una tradizione, di un campo e di un ruolo" 29 . Questa crisi poetica fa infatti crollare la distanza tra poeta e pubblico, cancella il valore dell'artisticità come tale, sostituendola con il concetto di poesia diffusa ${ }^{30}$. In quest'ottica possiamo capire meglio il senso del ritorno deangelisiano alla poetica di Baudelaire: I fiori del male, come affermò Walter Benjamin, è stato uno degli ultimi libri importanti di poesia ad aver avuto un successo di pubblico ${ }^{31}$. Il riferimento alla sua poetica diventa così una tappa imprescindibile per la ricostruzione del fondamento dei valori artistici, sognato da De Angelis.

Al livello formale, ciò che sembra essere uno strumento di difesa, nei confronti della cultura pop, è l'oscurità in cui De Angelis veste il suo linguaggio. Realizzatasi nell'immediatezza e intransitività nell'istante, la sua visione diventa fortemente ellittica e frammentaria, mentre il cortocircuito prodotto da sovraccarico delle tensioni solo a volte entra nelle linee figurative delle immagini che, come nota l'autore stesso, potrebbero sotto qualche aspetto assomigliare a rappresentazioni pittoriche di Edward Hopper. Tali unità di oggettiva presenza, prese nell'attimo di una situazione reale, si sciolgono però in modo istantaneo e quasi impercepibile, scivolando verso un groviglio di idee, sentimenti, angosce, realizzazioni poetiche di quadri di Franz Kline, Bacon o de Kooning. E così, i doveri, i tram, i cappotti, della poesia citata, si mescolano agli concetti sfocati e quasi irreali, alla pioggia e al vetro. L'io lirico con una precisione matematica scruta le cose, cercando di individuare il carattere e le dinamiche di ciò che sfugge fra di loro, ciò che "non può essere natura né storia", che si rispecchia nelle gocce che scorrono

28 G. Barberi Squarotti, op. cit., pp. 373-374.

29 A. Berardinelli (1975), "Effetti di deriva", [in:] Alfonso Berardinelli, Fabio Cordelli (a c. di) Il pubblico della poesia, Cosenza, Lerici, p. 16.

30 Cfr. G. Mazzoni, "Sulla storia sociale della poesia contemporanea in Italia", Ticontre. Teoria Testo Traduzione, 8 (2017), pp. 1-26.

31 Cfr. W. Benjamin, (1939-1940), "Su alcuni motivi in Baudelaire”, [in:] Giorgio Agamben, Barbara Chitussi e Clemens-Carl Härle (a c. di), Charles Baudelaire. Un poeta lirico nell'età del capitalismo avanzato, Vicenza, Neri Pozza, 2012, pp. 853-854. 
sulla finestra. Come risultato, la baudelairiana moltiplicazione di orizzonti viene portata all'ultimo limite. De Angelis sottomette la realtà ad infinite operazioni di calcolo: stringendo e focalizzando lo spazio, vuole sottrarla alla disgregazione. La rappresentazione del suo mondo inorganico, lontano dalla natura, viene concentrata al massimo e deprivata di ogni elemento epico; questa non racconta ma stringe il mondo ad una fissazione ossessiva del "sacro / rottame di ogni cosa" ${ }^{32}$, quello che non è più parte della realtà che rispecchi il tutto, ma ciò che porta lo squilibrio e, proprio in forza di esso acquisisce l'autonomia. "Interrompi il soprassalto", supplica il poeta, "riposa", "guarda", "accettala, la tua unica, la tua / gentile, lentissima morte"33. La tensione all'altrove rimane sempre vana e il carattere positivo della poesia non può che limitarsi ai miraggi di salvezza. Per questa ragione la verità del dire lirico si può riconoscere soltanto nell'atto di dimostrare la propria incapacità. Il mondo di De Angelis nasce quindi nel crollo, un crollo che diventa il centro della sua cosmoagonia. I versi sorgono dalla catastrofe, dal "precipitare del discorso poetico nella propria condanna sacrificale" ${ }^{34}$. Quella caduta diventa l'asse principale dei suoi componimenti che, pur risalendo al concetto del poeta veggente, non espongono i puri sentimenti o il clima nostalgico, non mettono in atto le dinamiche della poesia neo-orfica, ma operano all'interno della tensione tragico-sublime e dell'elemento dinamico che conduce alla disgregazione della pienezza.

La nostra ricerca di temi legati alla componente dell'italianità all'interno dell'opera di De Angelis, riporta anche alla categoria del mito e alle sue ascendenze letterarie. A tale proposito, dobbiamo fare un ricorso alla poetica di Cesare Pavese che propone una visione del mito inteso in quanto "schema di un fatto avvenuto una volta per tutte, [che] trae il suo valore da questa unicità assoluta che lo solleva fuori del tempo e lo consacra rivelazione" ${ }^{35}$. Tale aspetto ci permette di giustificare il carattere intransitivo delle parole di De Angelis. Queste operano infatti nelle dinamiche del continuo avvicendarsi di barlumi e ombre, di fracassi e silenzi che, in quanto due facce della stessa unità, lacerano il fondamento che la regge; risultano "simili alle note, estranee all'armonia, capaci solo di configgere, di urtarsi, producendo choc e tensioni continue" 36 . Lungo i suoi versi "tutte le cose sono un urto, non altro" ${ }^{37}$. Pavese, essendo uno dei più grandi esponenti della letteratura italiana del Novecento e una grande fonte

32 M. De Angelis, Tutte le poesie, p. 279.

33 Ibidem, p. 279.

34 A. Baldacci, op. cit., p. 93.

35 C. Pavese (2007), Feria d'agosto, Torino, Einaudi, p. 150.

36 A. Baldacci, op. cit., p. 92.

37 C. Pavese (1999), Dialoghi con Leucò, Torino, Einaudi, p. 21. 
d'ispirazione per De Angelis, non solo àncora la sua opera nell'orizzonte della letteratura nazionale, ma la apre anche alle dinamiche del pensiero nicciano. Grazie a questo fatto, il poeta milanese imposta la propria opera all'incrocio tra il dionisiaco e l'apollineo. I versi che salda sembrano rapiti dal furore delle origini, dalle sue forze drammatiche e furibonde. La voce dell'io si trova così di fronte a un temporale che trasmette "qualcosa che già c'era prima di noi" ${ }^{38}$. I luoghi raffigurati non sono più uno spazio impassibile, ma acquisiscono la vita, lo sguardo, la voce. Lo scopo del dire lirico risulta essere quello di chiamarli con il loro vero nome. Proprio per questo il registro dei componimenti concorda con la materia che rispecchia: non si contrappone alle cose, ma si scioglie nella loro natura. Il poeta, seguendo il pensiero di Pavese, cerca di aggrapparsi all'ultimo limite umano che gli è ancora rimasto, cioè all' istinto di rapprendersi in parole ${ }^{39}$. Il suo verso deve incidersi "all'interno di una struttura rigida, dove lo slancio dionisiaco è controbilanciato, o meglio domato e dominato da un gelo mentale che tende a tramutare l'eruzione della vita in pietra lavica, in 'delirio apollineo"'40.

I motivi che permettono di realizzare questa tensione vengono elaborati (con riferimenti principali a Maeterlinck e Leśmian) a partire dalla fiaba che, nel caso dell'autore milanese, resta segnata dalla "poetica dell'adolescenza" dell'autore, dal rapporto fra gioco e destino nonché dal tema della ragazzaguerriera, con richiami che vanno da Atalanta a Pentesilea. Tali nodi tematici si realizzano in quanto "ripetizioni di un gesto originario" ${ }^{41}$ che rivivono nei frammenti delle visioni e permettono di sfuggire al passare del tempo. Nella postfazione all'edizione di Tutte le poesie, De Angelis riconduce il significato della parola 'poesia' al "fare magico, artistico e mitico della sacra rappresentazione, del gioco e della festa" ${ }^{42}$. Anche nei suoi versi, come nota Stefano Verdino, "si produce quasi un sortilegio perché la fresatura tra la nitidezza del dettaglio e l'apertura visionaria dà al lettore un trasalimento per tanta sintetica forza e epiditticità del dire" ${ }^{43}$.

La precisione formale di De Angelis, "un'adesione millimetrica"44 al dettato poetico, il suo addirsi al tono del paesaggio, ma anche un continuo "lavoro di messa a fuoco" ${ }^{\prime 4}$, non smorzano il carattere visivo e spontaneo delle immagini,

38 M. De Angelis, Tutte le poesie, p. 407.

39 C. Pavese (1991), La letteratura americana e altri saggi, Torino, Einaudi, p. 287.

40 A. Baldacci, op. cit., p. 67.

41 E. Affinati (2008), Milo De Angelis e la sua nobile tristezza dell'adolescenza [in:] Milo De Angelis, Poesie, Milano, Mondadori, p. XI.

42 M. De Angelis, Tutte le poesie, p. 416.

43 S. Verdino (2017), Postfazione [in:] M. De Angelis, Tutte le poesie, p. 416.

44 M. De Angelis, Colloqui sulla poesia, p. 116.

45 Ibidem, p. 62. 
bensì creano fra loro una tensione interna. Tale carattere della sua produzione letteraria sembra fortemente segnato dall'impostazione di Franco Fortini, che De Angelis, come abbiamo precedentemente notato, considerava uno dei suoi maestri. E proprio grazie a Fortini il nostro poeta riesce a fondare, nella realtà degli anni Settanta, una linea capace di opporsi alla negazione dei versi lirici. Così viene aperto uno spazio poetico che De Angelis definisce come "tribunale delle parole $[\ldots]$ il luogo in cui tutti i poeti venivano chiamati a rispondere delle proprie pagine": "come se dalla riuscita della parola dipendesse il destino del mondo" 46 .

Come si è cercato di evidenziare, nel corso delle analisi, Milo De Angelis è un autore estremamente aperto agli influssi da parte del quadro artistico mondiale, con cui entra in un dialogo continuo. I suoi riferimenti vanno da Baudelaire a Dostoevskij, da Rimbaud a Lucrezio, da Maeterlinck a Leśmian, da Drieu La Rochelle a Bacon a De Kooning. Nondimeno il centro ispiratore, la calamita, dell'opera di De Angelis risiede all'interno dell'orizzonte italiano, ovvero nel paesaggio della sua Milano, periferica ed irreale, che più cambiamenti riporta, più rimane se stessa; nonché negli influssi del pensiero dei maestri letterari, che costituiscono il fondamento della sua poetica. Tra di essi ritroviamo Fortini, Luzi, Bigongiari, Leopardi, Montale, Pavese. Proprio quel ritorno, sia topografico che tematico e formale, ci porta nel luogo "dove eravamo già stati” e rivela l'italianità di quest'opera. Per finire il nostro discorso, sembra opportuno citare un frammento della poesia L'ago del ritorno, in cui possiamo ritrovare le dinamiche che finora si è provato ad esplicitare:

Tornano, vedi, ricomposte le visioni sono la prima e la penultima, i buoni tramonti di ogni cosa, di ogni cosa: riposa nell'unica durata, nel battito delle tangenziali e della mente, nel centro del buio, unantica rima mescolata alla vita, un calice sparso sul catrame ${ }^{47}$.

\section{Bibliografia}

Affinati, Eraldo (1996). Patto giurato. La poesia di Milo De Angelis, Pescara, Tracce Edizioni. Afribo, Andrea (2017). Poesia italiana postrema. Dal 1970 a oggi, Roma, Carocci.

Baldacci, Alessandro (2014). La necessita del tragico, Massa, Transeuropa.

Barberi Squarotti, Giorgio (1975). Introduzione a "L'idea centrale", Almanacco dello Specchio, 4, pp. 373-375.

46 Ibidem, p. 104.

47 M. De Angelis, Tutte le poesie, p. 279. 
Baudelaire, Charles (2008). I fiori del male, Venezia, Marsilio.

Benjamin, Walter (1939-1940). "Su alcuni motivi in Baudelaire", [in:] Giorgio Agamben, Barbara Chitussi e Clemens-Carl Härle (a c. di), Charles Baudelaire. Un poeta lirico nell'età del capitalismo avanzato, Vicenza, Neri Pozza, 2012.

Benjamin, Walter (1986). Parigi capitale del XIX secolo, Torino, Einaudi.

Berardinelli, Alfonso (1975). "Effetti di deriva", [in:] Alfonso Berardinelli, Fabio Cordelli (a c. di), Il pubblico della poesia, Cosenza, Lerici, 1975.

Berardinelli, Alfonso (1994). La poesia verso la prosa. Controversie sulla lirica moderna, Torino, Bollati Boringhieri.

Bonnefoy, Yves (2016). Il secolo di Baudelaire, Bergamo, Moretti\&Vitali.

Cucchi, Maurizio (1994). "Milo De Angelis", [in:] Maurizio Cucchi, Stefano Giovanardi (a c. di), Poeti italiani del secondo Novecento. 1945-1995, Milano, Mondadori.

De Angelis, Milo (1979). La corsa dei mantelli, Milano, Guanda.

De Angelis, Milo (1982). Poesia e destino, Bologna, Cappelli.

De Angelis, Milo (2008). Colloqui sulla poesia, Milano, La Vita Feice.

De Angelis, Milo (2008). Poesie, Milano, Mondadori.

De Angelis, Milo (2017). La parola data (interviste 2008-2016), Milano, Mimesis.

De Angelis, Milo (2017). Tutte le poesie, Milano, Mondadori.

Friedrich, Hugo (1971). La struttura della lirica moderna. Dalla metà del XIX alla metà del XX secolo, Milano, Garzanti.

Mazzoni, Guido "Sulla storia sociale della poesia contemporanea in Italia”, Ticontre. Teoria Testo Traduzione, 8 (2017), pp. 1-26.

Pavese, Cesare (1991). La letteratura americana e altri saggi, Torino, Einaudi.

Pavese, Cesare (1999). Dialoghi con Leucò, Torino, Einaudi.

Pavese, Cesare (2007). Feria d'agosto, Torino, Einaudi.

Pepe, Paola (1997). Tendenze e problemi nella poesia italiana dell'ultimo ventennio. Prefazione di Giordio Bàrberi Squarotti, Torino, Edizioni dell'Orso.

Prete, Antonio (2007). I fiori di Baudelaire, Roma, Donzelli. 